\title{
COMPUESTOS N-N Y DERIVACIÓN EN ESPAÑOL Y PORTUGUÉS BRASILEÑO: EN LA ENCRUCIJADA MORFOLOGÍA, SINTAXIS Y SEMÁNTICA*
}

\author{
Juana M. Liceras \\ University of Ottawa \& Universidad Nebrija \\ Rachel Klassen \\ Isabel Contro Castaldo \\ Faculty of Technology of São Paulo
}

\section{ResUmen}

En español, los afijos flexivos solamente se ligan al núcleo morfológico y semántico de los compuestos de dos sustantivos (N-N), lo cual implica que el plural de casa cuna o de hombre lobo es casaS cuna y hombreS lobo respectivamente mientras que *asa cunaS y *hombre loboS son agramaticales porque el afijo flexivo está ligado al modificador. Sin embargo, en el caso de los sufijos derivativos y, en concreto, del sufijo evaluativo diminutivo -ito, parece existir más flexibilidad ya que, para algunos hablantes, el sufijo puede tener abarque sobre todo el compuesto tanto si está ligado al núcleo (casITA cuna / hombrecITO lobo) como si está ligado al modificador (casa cunITA / hombre lobITO). A partir de una Tarea de Interpretación de Imágenes y de una Tarea de Juicios de Aceptabilidad que se administraron a un grupo de hablantes de espańol peninsular y de portugués brasileńo, se constata que el sufijo derivativo -ito se acepta con un grado significativamente mayor cuando va ligado al núcleo tal como sucede con el sufijo flexivo - s.

Palabras Clave: compuestos N-N, flexión, derivación, sufijos diminutivos, direccionalidad del núcleo.

\section{N-N COMPOUNDING AND DERIVATION IN SPANISH AND BRAZILIAN PORTUGUESE: AT THE CROSSROADS BETWEEN MORPHOLOGY, SYNTAX AND SEMANTICS}

\section{Abstract}

In Spanish, inflectional affixes attach to the morphological and semantic head of NounNoun (N-N) compounds, which implies that the plural of casa cuna or hombre lobo is casaS cuna and hombreS lobo respectively, while* casa cunaS and *hombre loboS are ungrammatical because the inflectional affix is attached to the modifier. However, there seems to be more flexibility in the case of derivational affixes, specifically evaluative diminutive affixes such as -ito, as for some native speakers, this suffix can have scope over the entire compound whether it is attached to the head (casITA cuna / hombrecITO lobo) or to the modifier (casa cunITA / hombre lobITO). In this article, data elicited via a Picture Interpretation Task and an Acceptability Judgment Task that were administered to a group of Peninsular Spanish speakers and to a group of Brazilian Portuguese speakers, show that derivative affix -ito is significantly more acceptable when attached to the head of the N-N compound, as is the case with inflectional affix $-s$.

KEYwORDs: N-N compounds, inflection, derivation, diminutive affixes, headedness.

DOI: https://doi.org/10.25145/j.refiull.2020.40.07

Revista de Filología, 40; enero 2020, pp. 125-145; ISSN: e-2530-8548 


\section{INTRODUCCIÓN}

Nuestro interés por los compuestos N-N comenzó al final de los 90 y en el contexto del llamado Parámetro de los Compuestos (Snyder, 1995), al cual daban nombre estos sustantivos. La propuesta era que en torno a la opción positiva de este parámetro se agrupaban las lenguas en las que los compuestos N-N eran productivos (el inglés o las lenguas germánicas) porque sus categorías léxicas o sustantivas tienen el rasgo [+afijo], mientras que las lenguas en las que esos compuestos no son productivos (el español y las lenguas romances) son las de categorías sustantivas con el rasgo [-afijo]. Lo primero que nos propusimos investigar fue el hecho de que, si bien estos compuestos no eran productivos, sí que era obvio que los hispanohablantes tenían intuiciones claras sobre cómo se formaban y podían interpretar compuestos N-N que no estaban atestiguados o no eran frecuentes. De hecho, y dado que la teoría paramétrica está ligada a la variación que existe entre las lenguas y a la adquisición del lenguaje (Chomsky, 1981; Chomsky \& Lasnik, 1993; Snyder, 1995, 2001, 2007), la forma en la que los nativos abordan estos compuestos en distintas tareas experimentales y sus intuiciones sobre estas construcciones es una fuente importante de información sobre su conocimiento de la lengua, en concreto de la formación de palabras, de la direccionalidad del núcleo y, por supuesto, sobre qué es lo que ha de adquirir o suprimir de una de las lenguas el que adquiere dos lenguas (o más) de forma simultánea o secuencial, como niño o como adulto.

De esos temas, sobre todo del español como lengua objeto con el inglés, el francés y el finés como lenguas maternas, han tratado distintos autores, entre ellos, Liceras \& Díaz (2000); Liceras, Díaz \& Salomaa-Robertson (2002); Pomerleau (2001) o Salomaa-Robertson (2000).

Nosotros vamos a ocuparnos de la relación entre los compuestos y los sufijos derivativos (mujercITA pulpo / mujer pulpITA), aunque siempre comparándolos con los flexivos (mujerES pulpo / *pulpo mujerES), y vamos a centrarnos en cómo conceptualizan y procesan los compuestos con sufijos derivativos (y flexivos) los adultos hablantes de español peninsular (ESP) y de portugués brasileño (PB) como lenguas maternas. Como ambas lenguas son de núcleo a la izquierda, podemos argumentar que las propuestas que se han hecho para la morfología flexiva del ESP se pueden aplicar al PB.

* Queremos dar las gracias a todos los participantes, tanto de España como de Brasil, y a los colegas del UVALAL [http://albergueweb1.uva.es/uvalal/], especialmente a la directora, Raquel Fernández Fuertes, por su apoyo académico y logístico. Este trabajo ha sido posible gracias a los fondos de investigación que hemos obtenido de (i) la Facultad de Artes de la Universidad de Ottawa a J. M. Liceras, (ii) la beca de Excelencia de la Universidad de Ottawa y la beca del Consejo de las Humanidades y Ciencias Sociales (SSHRC) a R. Klassen, (iii) la beca postdoctoral Marie Skłodowska-Curie a R. Klassen y (iv) la beca PDSE -Programa Doctorado Sándwich en el Exterior- de la CAPES -Coordinación de Perfeccionamiento de Personal de Nivel Superior- Brasil a I. Contro-Castaldo. 
También queremos hacer especial hincapié en que el análisis y la descripción de estos compuestos son necesarios para investigar cómo se representa la direccionalidad del núcleo o el género en la mente del hablante nativo, del bilingüe y del hablante no nativo. Para llevar a cabo esa investigación, hemos puesto la lingüística formal al servicio de la psicolingüística, ya que hemos utilizado dos pruebas experimentales, una Prueba de Interpretación de Imágenes (PIT) y una Prueba de Juicios de Aceptabilidad (AJT) ${ }^{1}$, dirigidas a determinar si el núcleo morfológico y semántico se representa en la mente de los hablantes de ESP y de PB como propone Zwicky (1998) o como propone Cinque (2005).

Queremos discutir una problemática que, hasta donde sabemos, no se ha tratado en las gramáticas al uso y no se ha abordado desde la psicolingüística o la adquisición salvo en Liceras \& Klassen (2016 y 2019) y en Klassen, Contro Castaldo \& Liceras (2018). El estudio que presentamos aquí nos permite abordar el tema de cómo se representan estos compuestos (y sus propiedades morfosintácticas) en la mente de los nativos de las dos lenguas que nos ocupan, discutir esos aspectos morfosintácticos y plantearnos qué aspectos semánticos pueden formar parte de esa representación mental configurando los juicios que van a emitir y las imágenes que van a elegir como representativas de los compuestos. Nos permite además aportar datos e información que puede serle de utilidad al morfólogo y al psicolingüista de forma directa y al profesor de lengua, de forma indirecta.

Creemos que este tema del que nos ocupamos aquí es clave en lo que se refiere a dar cuenta de cómo los afijos flexivos marcan la direccionalidad del núcleo (la marca de plural irá en el núcleo y no en el modificador, de ahí la gramaticalidad de dos perroS policía frente a la agramaticalidad de *dos perro policíaS). Lo mismo sucede también en portugués, de forma que el sustantivo dois cáeS-polícia es gramatical, mientras que el sustantivo *dois cão-polícia es agramatical. Dejamos claro también que la aceptabilidad de compuestos en los que el sufijo derivativo se liga al modificador es mayor que la de los compuestos en los que es el sufijo flexivo el que se liga al modificador y que el doble ligamiento (al núcleo y al modificador), en el caso de la flexión de plural (hombreS loboS, mujerES pulpoS o cartaS bombaS), es claramente más aceptable en PB que en ESP y que, en el caso de la derivación, el doble ligamiento (hombrecITO lobITO, mujercITA lobITA o cartITA bombITA) recibe una interpretación por parte de los hablantes nativos pero no parece ser aceptable en ninguna de las dos lenguas.

${ }^{1}$ Hemos decidido mantener las siglas inglesas de los títulos de las tareas, PIT para Picture Interpretation Task y AJT para Acceptability Judgments Task, porque su uso es más frecuente que el de las siglas en español. 


\section{LOS COMPUESTOS N-N Y LA FLEXIÓN}

Vamos a presentar primero cómo funciona la flexión de número en los compuestos N-N y, para representar los compuestos a que hacemos referencia, vamos a utilizar los dibujos que Terhi Salomaa-Robertson creó para nosotros y que han sido y siguen siendo un material importante en nuestros trabajos de investigación ${ }^{2}$.

Lo que parece indiscutible es que podemos interpretar los dibujos de (1)a y (2)a como un perro policía y una mujer pulpo en ESP y um cão-polícia y uma mulherpolvo en $\mathrm{PB}$ y que el plural se forma ligando el sufijo flexivo $-s$ al núcleo del compuesto -el sustantivo que se sitúa a la izquierda-, como se muestra en (1)b.

(1)a

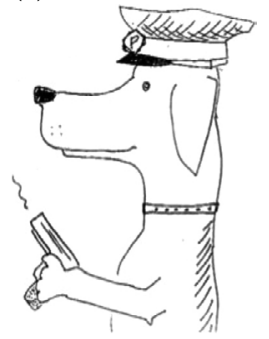

un perro policía

um cão-polícia

a dog police

'a police dog'

(2)a

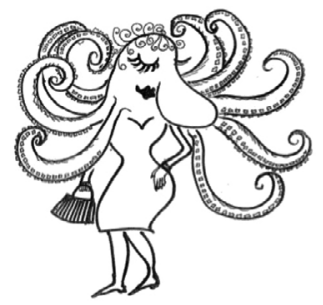

una mujer pulpo uma mulher-polvo a woman octopus 'an octopus woman' (1)b
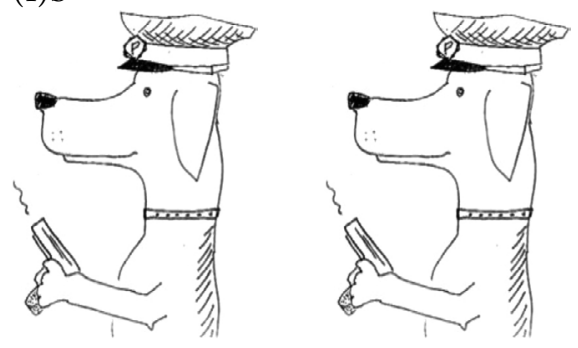

dos perroS policía / *dos perro policías dois cãeS-polícia / *dois cão-políciaS dois cãeS-políciaS two dogs police 'two police $\operatorname{dog} S$ '

(2)b
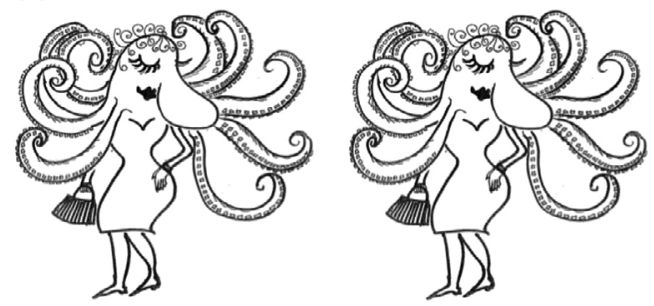

dos mujerES pulpo / *dos mujer pulpoS duas mulherES-polvo / *duas mulher-polvoS duas mulherES-polvoS two women octopus 'two octopus womEN'

2 Terhi Saloma-Robertson es la autora de estos dibujos, los cuales le fueron donados en exclusiva a nuestro Language Acquisition Research Lab [http://artsites.uottawa.ca/larlab/]. 
La traducción al inglés para el mismo dibujo o la misma figura nos deja claro que el ESP y el PB son lenguas de núcleo a la izquierda mientras que el inglés es una lengua de núcleo a la derecha, ya que la secuencia de los sustantivos en inglés no coincide con la del ESP y el PB y, por lo tanto, el $\mathrm{N}$ al que se liga el afijo flexivo es el de la derecha en inglés ( $\operatorname{dog} S$, womEN) y el de la izquierda en ESP (perroS, mujerES) y $\mathrm{PB}$ (cäeS, mulherES).

La morfosintaxis de los compuestos funciona igual cuando se refieren a un objeto inanimado como en el caso de barcoS pirata, muebleS bar o casaS cuna, o de los dibujos de (3).

(3) a

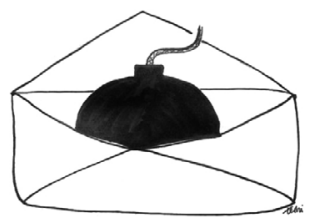

una carta bomba uma carta-bomba a letter bomb 'a letter bomb'
(3) $\mathrm{b}$
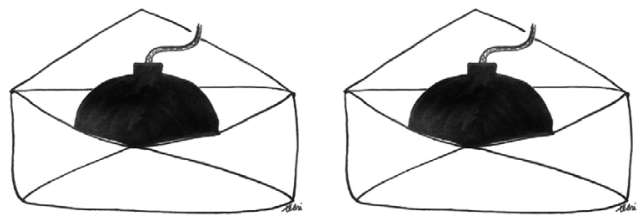

dos cartaS bomba / *dos carta bombaS duas cartaS-bomba / *duas carta-bombaS duas cartaS-bombaS two letter bombs 'two letter bombS'

Lo que vemos en (3)b es que el sufijo flexivo de plural se une también al núcleo y, cuando hablamos de núcleo, hablamos de que en todos los casos que nos ocupan los afijos flexivos marcan el núcleo morfológico y este se corresponde con el semántico. Es decir, y siguiendo a Allen (1978), la definición de carta bomba sería "una carta que... tiene una bomba dentro», por ejemplo, es decir CARTA es el núcleo morfológico (lleva la marca de plural) y semántico (el compuesto se define a partir del sustantivo que está a la izquierda). Pero si comparamos este ejemplo con los de (1) y (2), vemos que el orden de los sustantivos de la versión inglesa es el mismo que el de las versiones del ESP y el PB, es decir, es letter bomb y no bomb letter. Y sin embargo, el plural de letter bomb, como vemos en la leyenda de (3)b, es 'letter bombS', lo cual muestra que en inglés el sufijo flexivo plural va también en el núcleo, y el núcleo es el sustantivo que se sitúa a la derecha y no a la izquierda como en ESP y en PB. Esto implica que el núcleo semántico es bomba y que ese objeto se define como «una bomba que... está dentro de una carta». En otras palabras, y si bien parece que el ESP ha adoptado la traducción del inglés manteniendo el orden de los dos sustantivos, lo que en realidad ha sucedido es que en español se ha ligado el sufijo flexivo al sustantivo que está a la izquierda y eso ha llevado a que se reconceptualice la semántica del compuesto y, por lo tanto, a que se defina a partir del sustantivo que se sitúa a la izquierda, es decir, como «una carta que... tiene una bomba dentro", tal como hemos indicado antes. Así lo interpretan también los hablantes de PB aunque este compuesto tampoco sea frecuente en esa lengua. 


\section{LOS COMPUESTOS N-N Y LA DERIVACIÓN}

Una pregunta que Liceras \& Klassen (2016 y 2019) se hacen para el espanól es si en el caso de la morfología derivativa es posible ligar el sufijo diminutivo (u otros sufijos evaluativos) al modificador. En otras palabras, se trata de determinar si el compuesto carta bombITA, en el que el afijo diminutivo está unido al modificador y no al núcleo, es gramatical e interpretable y, si lo es, si ese sufijo tiene abarque sobre todo el compuesto de forma que carta bombITA sea el equivalente de cartITA bomba, es decir, en los dos casos una versión pequeña de una carta bomba.

Independientemente de que se considere que los sufijos evaluativos forman parte de la derivación, lo que está claro para nosotros es que son diferentes de la flexión, y no hay duda de que lo son en lo que se refiere a la semántica porque mientras el plural tiene un significado establecido, el valor de tamaño pequeño que asigna el diminutivo podemos decir que es la interpretación por defecto, pero también hay otras acepciones como la de 'agradable', 'gracioso', 'mono', 'simpático', etc.

Y puesto que tenemos las imágenes y podemos 'jugar' con ellas, si se acepta esa posición del afijo, ¿̇tiene la misma interpretación que cuando se liga al núcleo? Es decir, ¿pueden interpretarse (4)b y (5)b como la representación de (i) y de (ii) en cada caso?

$(4) \mathrm{a}$

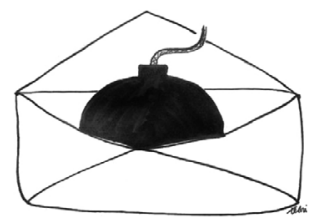

carta bomba
$(4) \mathrm{b}$

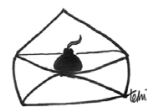

(i) cartITA bomba / cartINHA-bomba

(ii) carta bombITA / carta-bombINHA

$(5) \mathrm{b}$
(5) a

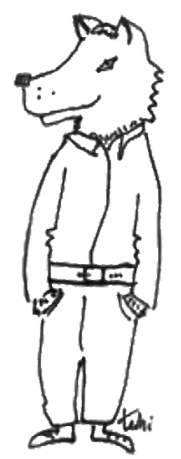

hombre lobo

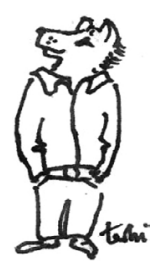

(i) hombrecITO lobo / homenzINHO-lobo

(ii) hombre lobITO / homem-lob $\underline{\mathrm{INHO}}$ 
También nos preguntamos si cabe la posibilidad de que el sufijo derivativo tenga abarque sobre el núcleo o solamente sobre el modificador, según vaya ligado a uno u otro, como se representa en (6) y (7) respectivamente.

(6) a

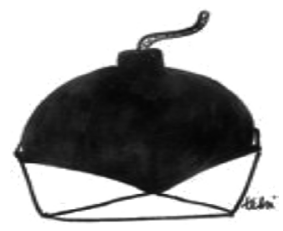

cartITA bomba cartINHA-bomba

(7)a

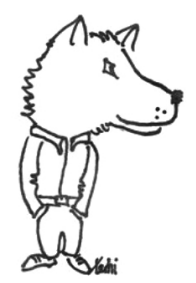

hombrecITO lobo homenzINHO-lobo (6)b

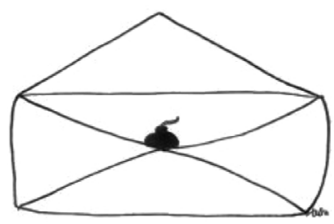

carta bombITA carta-bombINHA

(7)b

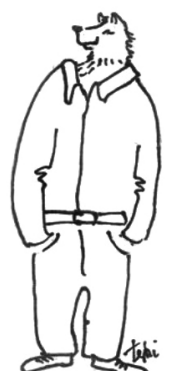

hombre lobITO homem-lobINHO

Finalmente, cabe preguntarnos si, como es el caso con la doble sufijación del plural, que es bastante más aceptable en PB (duas cartaS-bombaS) que en ESP (?dos cartaS bombaS), también se aceptará la doble sufijación en el caso de la morfología derivativa en PB (uma cartINHA-bombINHA) y menos en ESP (una cartITA bombITA). Y si se acepta o se interpreta, ¿se conceptualiza como una versión pequeña del compuesto sin afijo, es decir, como el compuesto que lleva la flexión en el núcleo, las imágenes de (4)b y (5)b que repetimos aquí como (8)a y (8)b con la leyenda de doble afijación?

(8)a

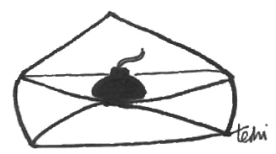

cartITA bombITA cartINHA-bombINHA (8)b

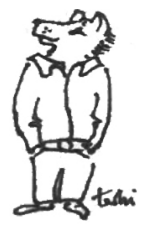

hombrecITO lobITO homenzINHO-lobINHO 
En otras palabras, lo que proponemos es que cuando el sufijo derivativo se liga tanto al núcleo como al modificador, el sustantivo se conceptualiza igual que si el sufijo derivativo se liga solamente al núcleo, de forma que el resultado -(8)a y (8)b- es una versión pequeña a escala, es decir, equilibrada, de los sustantivos neutros de (4)a y (5)a, como lo es (4)b y (5)b.

\section{EL ABARQUE DEL SUFIJO EVALUATIVO}

Si bien parece haber consenso entre los lingüistas en que el sufijo flexivo se sitúa en el núcleo del compuesto y si no es así el resultado es agramatical, como hemos visto en (1)b, (2)b y (3)b, el sufijo -ito ligado al modificador como en (6)b y (7)b no produce ese mismo rechazo. Sin embargo, la propuesta de Zwicky (1998) es que tanto en el caso de la morfología flexiva como en el de la derivativa, el sufijo solamente ha de ligarse al núcleo del compuesto, lo cual quiere decir que para este autor, los compuestos con afijo flexivo en el modificador como *hombre lobITO / *homem-lobINHO o *carta bombITA / *arta-bombINHA son agramaticales. Esta propuesta no contempla, pues, la posibilidad de que estos compuestos sean interpretables aunque no se consideren sinónimos de los que llevan al sufijo en el núcleo y por tanto tengan abarque sobre todo el compuesto, sino con abarque solamente sobre el modificador, como nos preguntábamos en (6)b y (7)b. Tampoco contempla la posibilidad de que el sufijo en el núcleo pueda tener abarque solo sobre el núcleo como se representaba en (6) a y (7)a. Sin embargo, creemos que los niveles de aceptabilidad de las oraciones que figuran en (9) y (10) no son iguales.

$\begin{array}{lll}(9) \mathrm{a} & * / ? ? & \text { Es una cartITA bomba muy grande } \\ (9) \mathrm{b} & ? ? & \text { Es una carta bombITA muy grande } \\ (9) \mathrm{c} & \text { ?? } & \text { Es una cartITA bombITA muy grande } \\ & & \\ (10) \mathrm{a} & * / ? ? & \text { Es un hombrecITO lobo enorme } \\ (10) \mathrm{b} & ? ? & \text { Es un hombre lobITO enorme } \\ (10) \mathrm{c} & ? ? & \text { Es un hombrecITO lobITO enorme }\end{array}$

En (9)a y en (10)a tachamos de altamente agramaticales esas oraciones por la contradicción que introducen los adjetivos muy grande y enorme. Esto quiere decir que al sufijo -ito se le otorga el valor por defecto, el de tamaño pequeño. Si este fuera el caso en (9)b y (10)b y de (9)c y (10)c, también tendríamos que considerarlas altamente agramaticales. Ahora bien, lo que queremos proponer es que estos últimos ejemplos, tanto el de los compuestos que llevan el sufijo en el modificador como en los compuestos con fijación doble, el valor afectivo de -ito podría atenuar o eclipsar el valor por defecto, lo cual llevaría a que los adjetivos muy grande y enorme no entraran en contradicción con el valor que otorga el sufijo porque no se refiere al tamaño sino a la calidad de 'agradable; 'mono' o 'simpático' a que hemos aludido con anterioridad. 
Pero queremos precisar que, tanto si se adopta el valor por defecto del sufijo como si no, hay hablantes que están dispuestos a aceptar que cuando el sufijo se liga al modificador tiene abarque sobre todo el compuesto, es decir, que, para ellos, casITA cuna y casa cunITA son variantes de un mismo compuesto. La propuesta de Cinque (2005) con respecto al orden prenominal y postnominal de los adjetivos permite dar cuenta de que el modificador tenga abarque sobre todo el sintagma nominal, con lo cual esa opción gramatical estaría avalada desde un punto de vista sintáctico. Pero, en el caso de que no sea una opción generalizada, también podríamos plantearlo como una manifestación de 'deponencia' en la morfosintaxis, adoptando la propuesta de Bermúdez-Otero (2007). Es decir, al igual que en lenguas como el latín hay verbos, los llamados deponentes, que tienen morfología pasiva y significado activo como en el ejemplo de (11) que da el propio Bermúdez-Otero, podríamos proponer que en estos compuestos con afijo en el modificador el orden de palabras es el típico de la lengua que es de núcleo a la derecha y que ese sustantivo es el responsable de la semántica del compuesto y que, sin embargo, la morfología no se ajusta a ese patrón.

\begin{tabular}{llll} 
Nempe & patrem & \multicolumn{1}{c}{ sequ-untur } & liber-i \\
Por supuesto & padre $_{\text {(acus.sing) }}$ & sigue $_{\text {(3p.pl.pres.ind.pasiva) }}$ & hijos $_{\text {(nom.pl.) }}$ \\
'Por supuesto el padre sigue a los hijos' & &
\end{tabular}

[Livy, At urbe condita, IV, 4,11]

En esta línea, el hecho de que el sufijo derivativo ligado al modificador tenga abarque sobre todo el compuesto podría también considerarse un caso de 'deponencia' morfosintáctica, ya que hay conflicto entre la posición morfológica del sufijo (en el sustantivo situado a la derecha) y la interpretación de la direccionalidad del núcleo en el plano semántico (la misma que cuando el sufijo se liga al sustantivo situado a la izquierda).

\section{ESTUDIO EMPÍRICO}

Con objeto de investigar si la derivación en los compuestos del ESP y del $\mathrm{PB}$ tiene el mismo estatus que la flexión y si hay diferencias entre las dos lenguas, independientemente de que se comporten de la misma forma en lo que se refiere a la direccionalidad del núcleo (a la izquierda), hemos llevado a cabo un estudio empírico para el que se han diseñado las dos pruebas experimentales que se han mencionado, la PIT, que se administró en primer lugar, y la AJT, que se administró después y a grupos distintos de participantes. Ambas pruebas se administraron en las dos lenguas a los respectivos grupos de hablantes nativos, como sigue: (i) PIT a 51 nativos de ESP y a 66 nativos de PB y (ii) AJT a 66 nativos de ESP y a 59 nativos de $\mathrm{PB}$. Hemos de precisar también que nos acogemos al planteamiento que comparten muchos lingüistas según el cual la gramaticalidad la propone o establece el lingüista a partir del modelo que adopta y que da cuenta de lo que generan las reglas 
y principios que estipula ese modelo. La aceptabilidad, por su parte, es patrimonio del hablante nativo y puede no coincidir cien por cien con la gramaticalidad.

\subsection{Preguntas de investigación}

Las preguntas de investigación que nos proponemos contestar y las hipótesis que queremos testar con estas pruebas son las siguientes:

\#1. ¿Tiene la morfología derivativa el mismo estatus que la flexiva en la mente de los hablantes nativos de ESP y PB?

Hipótesis. Si la morfología derivativa es como la flexiva, el sustantivo N-N con el afijo en el núcleo tendrá abarque sobre todo el compuesto y el sustantivo N-N con el afijo en el modificador, que en principio debería ser rechazado como se muestra en (13), y que es lo que sucede en el caso de la flexión como hemos visto en el segundo apartado y como vemos en (12), en la PIT se manifestará en que el sufijo derivativo en el modificador del sustantivo $\mathrm{N}-\mathrm{N}$ no tenga abarque sobre todo el compuesto sino que, si se interpreta, tendrá solamente abarque sobre el modificador.

\section{hombreS lobo / *hombre loboS}

(13) hombrecITO lobo / ??hombre lobITO

Si al contrario de lo que sucede con la flexión, el sufijo en el modificador se interpreta como el sufijo en el núcleo, entonces la elección de figura coincidirá en los dos casos: es decir, se va a elegir la opción equilibrada pequeña del compuesto 'neutro', el que no tiene afijo. En el caso de la AJT, la mayor aceptación será para los casos de compuestos neutros (sin afijo) y de compuestos con el sufijo en el núcleo.

\#2. ¿Cómo se interpretan los compuestos con doble sufijación como el de (14)?

$$
\text { hombrecITO lobITO }
$$

Hipótesis. En la PIT, que no da la opción del rechazo sino de interpretación de las imágenes, se espera que estos compuestos se interpreten como los compuestos cuya sufijación tiene abarque sobre la totalidad del sustantivo, es decir, igual que los compuestos cuya sufijación va en el núcleo. En el caso de la AJT, se espera que esta opción se rechace si, como sucede en español, el índice de aceptación de la doble flexión de plural es bajo y que se acepte si, como sucede en portugués, ese índice de aceptación es alto. 


\subsection{Prueba de Interpretación de Imágenes: ESP}

\subsubsection{Participantes PIT: ESP}

Un grupo de 51 hablantes de español peninsular, todos adultos y con educación universitaria, llevaron a cabo la prueba que se describe a continuación.

\subsubsection{Diseño de la prueba experimental PIT (ESP)}

La prueba consistía en seleccionar el dibujo que mejor representara a los compuestos que se presentaban de forma escrita, como se muestra en (15). Se alternaban los compuestos de forma que las cuatro opciones (hombre lobo, hombrecito lobo, hombre lobito y hombrecito lobito) aparecieran con orden aleatorio con los 16 ítems experimentales (compuestos) que se incluyeron, y en la presentación de los dibujos las cuatro opciones también aparecían unas veces en un orden y otras en otro (por ejemplo, se alternaba de forma que primero aparecía la versión neutral, o la figura pequeńa equilibrada, o una de las dos que muestran desequilibrio entre los dos elementos del compuesto y luego aparecían en el orden opuesto, etc.). Es decir, la secuencia de imágenes de (15) a veces aparecía con la segunda imagen o la tercera o la cuarta en primer lugar y así se iba alternando.

HOMBRECITO LOBO
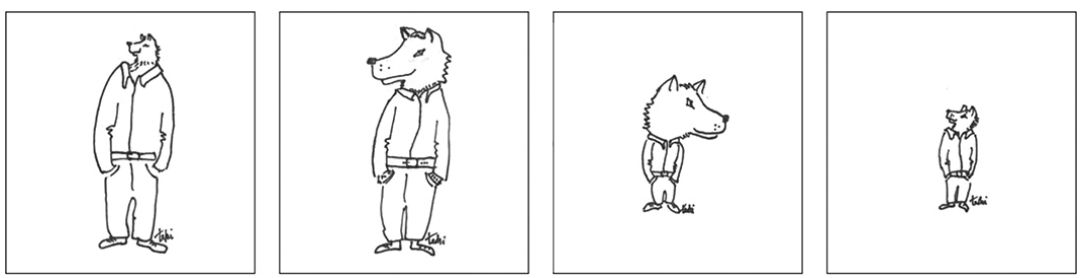

De los 16 ítems experimentales, ocho eran compuestos con el rasgo [+animado] y 8 con el rasgo [-animado]. Además, había 16 distractores en los que se proporcionaba un sustantivo seguido de un adjetivo como en el ejemplo de (16).
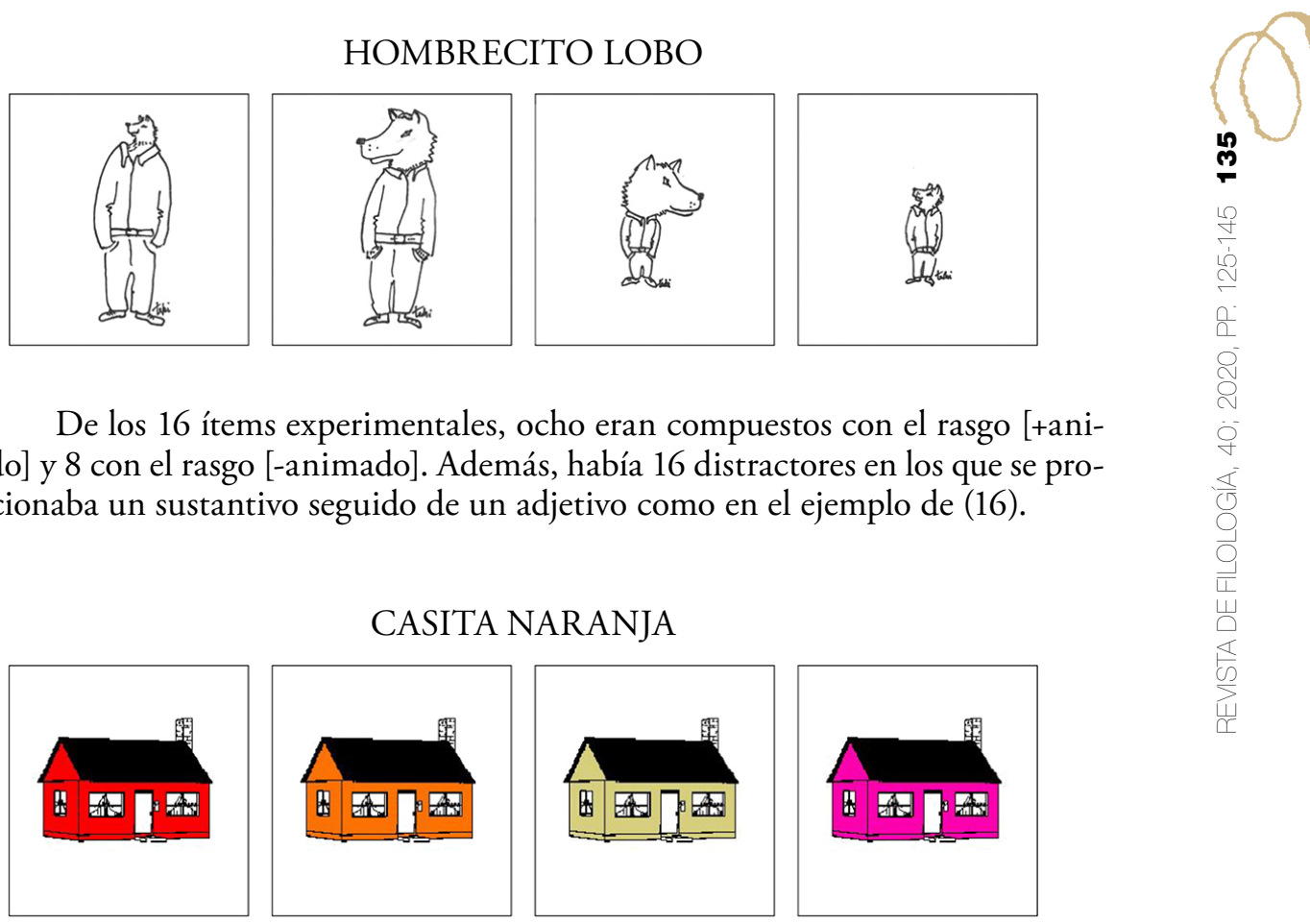
Para la codificación de datos, los dibujos se clasificaron como se indica en (17): (17)

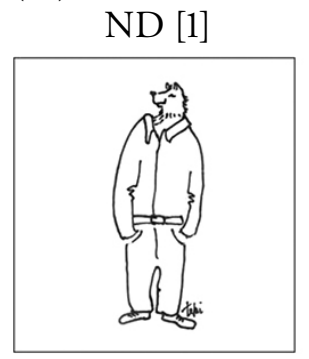

- NN

- $\mathrm{DN}$

- ND

- $\mathrm{DD}$
Ns sin afijo

afijo derivativo en el núcleo afijo diminutivo en el modificador afijo diminutivo en los dos
NN [2]

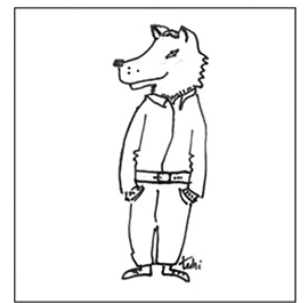

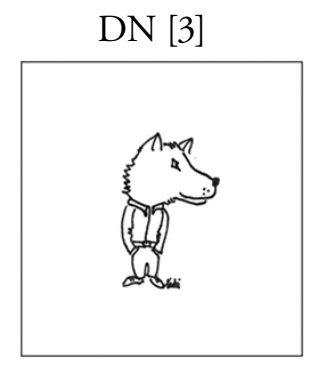

hombre lobo

hombrecITO lobo

hombre lobITO

hombrecITO lobITO
$\mathrm{DD}[4]$

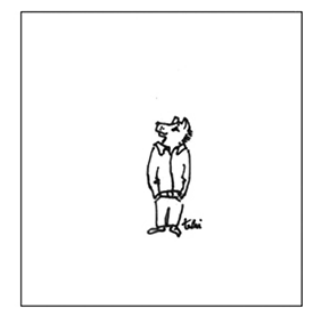

[2]

[4]

[1] o [4]

[4]

De acuerdo con nuestras hipótesis, se esperaba que para la opción sustantivo DN eligieran la imagen [4]. Para la opción sustantivo ND, se esperaba que eligieran la imagen [1] si no le dan al sufijo abarque sobre todo el compuesto y la opción [4] si se lo dan, es decir, si la representación DN y ND es la misma. La imagen [3] no se esperaba que la eligieran los hablantes nativos, puesto que supone que el sufijo en el núcleo no tenga abarque sobre todo el compuesto (lo que lleva a elegir la imagen [4]) sino solamente sobre el núcleo. Por lo que se refiere a la opción con doble sufijación, la DD, se esperaba que eligieran la imagen [4].

\subsubsection{Resultados PIT (ESP)}

Las opciones que eligieron los hablantes de ESP L1 se resumen en el gráfico 1.

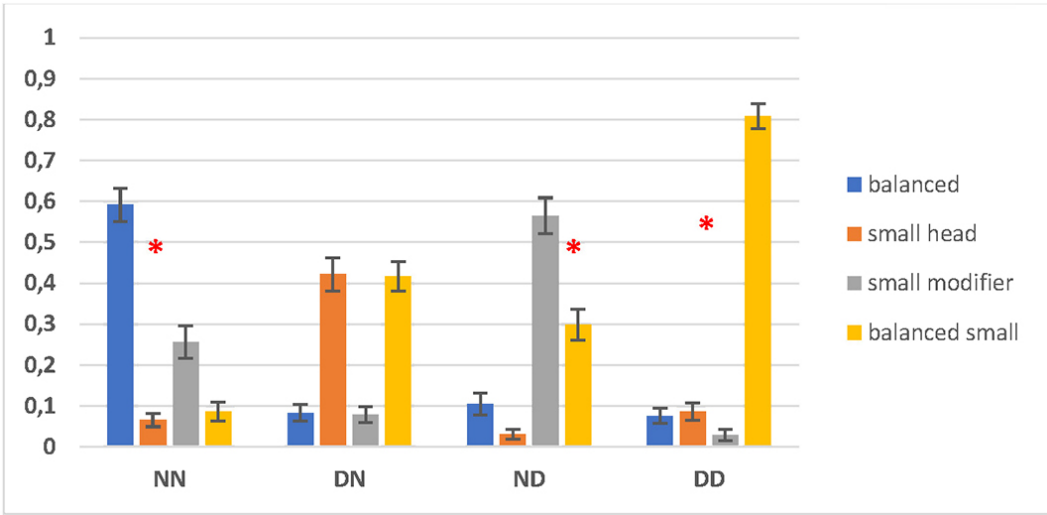

Gráfico 1. ESP L1: Prueba de Interpretación de Imágenes.

- NN equilibrado

- DN núcleo pequeño

- ND modificador pequeño

- DD pequeńo equilibrado 
Lo que nos muestra este gráfico es que cuando el compuesto 'neutral' (NN) se interpreta como la imagen en la que las dos partes del compuesto están equilibradas, la preferencia por esta opción, como muestra el asterisco rojo, es estadísticamente significativa. En el caso de los compuestos DN, con el diminutivo en el núcleo, esperábamos una preferencia significativa por la imagen en la que las dos partes del compuesto están equilibradas, pero es considerablemente más pequeña que la 'neutral', lo que nos encontramos es un empate entre esa imagen (la columna amarilla) y la imagen en la que solo el núcleo es pequeño (la columna naranja), es decir, entre las dos imágenes que reproducimos en (18).

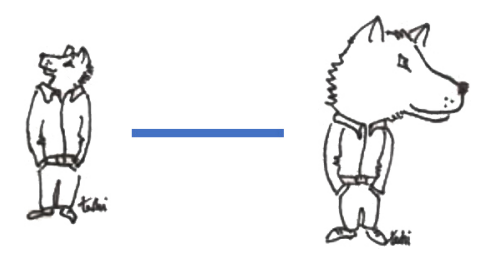

Nuestra interpretación de este resultado es que, aunque hayan elegido el dibujo de la derecha, los participantes interpretan el compuesto como una versión pequeña del compuesto 'neutral', si bien eligen el dibujo en que solamente el núcleo es pequeño porque es una figura pequeña y 'simpática'. También es posible que hayan 'aprendido' del espíritu de los dibujos de abarque sobre el núcleo y no sobre todo el compuesto. Es decir, es muy posible que la prueba en sí condicione las opciones de los hablantes más allá de sus intuiciones sobre los compuestos.

En cuanto al compuesto ND con el diminutivo en el modificador, la opción estadísticamente significativa es la que representa que el diminutivo solamente tiene abarque sobre el modificador (la columna gris), si bien algunos hablantes eligen la imagen con el hombre lobo pequeño y equilibrado (la columna amarilla). Finalmente, se confirma que, en el caso de la doble sufijación (el compuesto DD), y al verse obligados a seleccionar una imagen, los hablantes de ESP eligen al hombre lobo pequeño equilibrado (la columna amarilla) de forma estadísticamente significativa.

En el trabajo de Liceras \& Klassen (2019) nos preguntábamos si el sufijo -ito era "promiscuo" (podía ir ligado indistintamente al núcleo o al modificador) y la contestación a esa pregunta dependía de que se probara que los participantes relacionaban ambos sustantivos con la misma imagen (la versión pequeña del compuesto 'neutral'). Estos datos muestran que los hablantes de ESP L1 tratan la adjunción al núcleo y al modificador de forma diferente y esto nos lleva a argumentar que el hecho de que la teoría esté abierta a que la adjunción del sufijo al modificador tenga abarque sobre todo el compuesto no parece que sea una opción relevante para estos hablantes. 


\subsection{Prueba de Interpretación de Imágenes: PB}

Con esta prueba vamos a ser capaces de constatar si los hablantes de PB L1 se representan los compuestos con sufijo derivativo como los hablantes de ESP. Es decir, vamos a contestar a las dos preguntas de investigación que se formularon en 4.1. pero ahora en relación con el PB.

\subsubsection{Participantes PIT PB}

Esta prueba les fue administrada a 66 hablantes nativos de $\mathrm{PB}$, todos ellos residentes en Sao Paulo y con estudios universitarios.

\subsubsection{Diseño de la prueba experimental PIT (PB)}

Es la versión PB de la prueba de ESP que se describe en 4.2.2.

\subsubsection{Resultados PIT (PB)}

Los hablantes de PB, al igual que los hablantes de ESP, eligen, de forma estadísticamente significativa (como muestra el gráfico 2), la imagen del compuesto cuyas dos partes tienen un tamaño equilibrado cuando el sustantivo no tiene afijos (la columna azul en NN). Sin embargo, cuando el sustantivo tiene el sufijo en el núcleo (DN) no eligen la versión pequeña y equilibrada de la imagen sin sufijos, sino la imagen que representa un núcleo pequeño, la que está a la derecha en (18). No nos queda claro si prefieren esta imagen porque no consideran que el diminutivo en el núcleo tiene abarque sobre todo el compuesto (lo interpretan como la versión pequeña equilibrada del 'neutral' equilibrado) o porque les parece más 'graciosa' (dan al diminutivo la interpretación 'especial' de 'gracioso', 'simpático' y no lo conceptualizan como reducción de tamaño), independientemente de la relación núcleo-modificador en lo que respecta a la falta de equilibrio entre las dos partes del compuesto, que es obvia en el dibujo de la derecha de (18). 


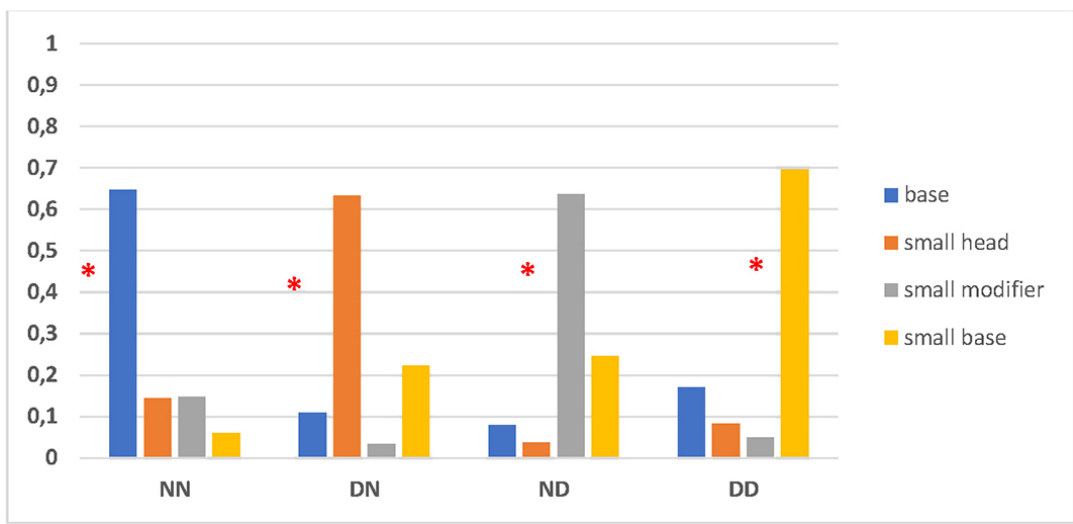

Gráfico 2. PB L1. Prueba de Interpretación de Imágenes.

- NN equilibrado

- DN núcleo pequeño

- ND modificador pequeño

- DD pequeño equilibrado

En el caso de los compuestos ND, la elección es la misma que la de los hablantes de ESP: la imagen con el modificador pequeño que vemos en (19).

(19)
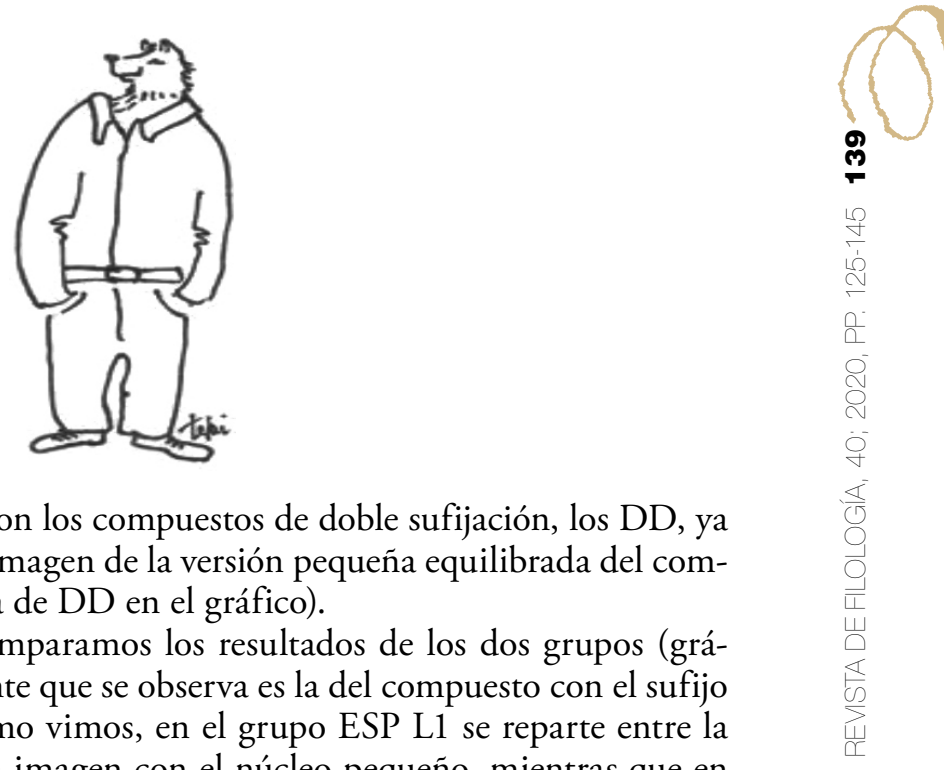
imagen pequeña equilibrada y la imagen con el núcleo pequeño, mientras que en PB L1 la mayor elección de esta segunda imagen es estadísticamente significativa. 

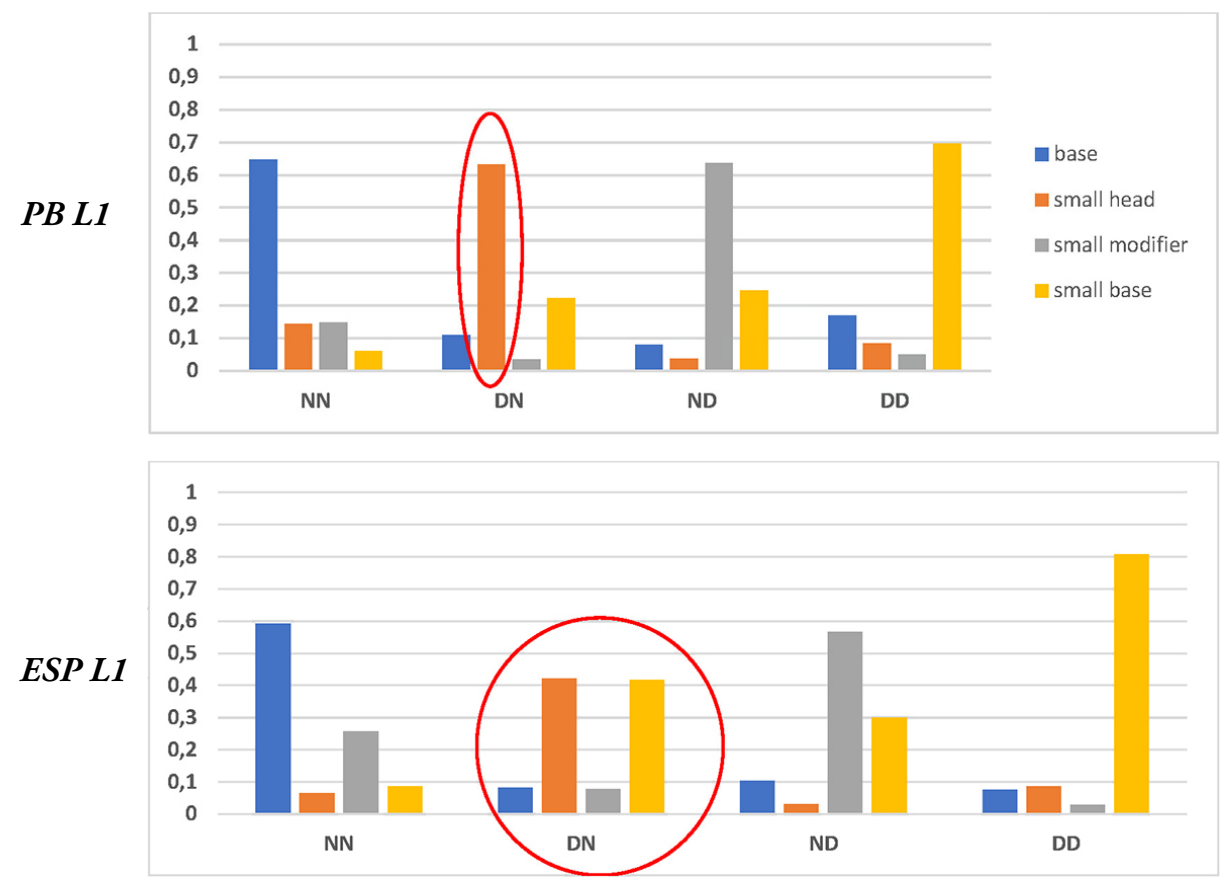

Gráfico 3. Prueba de Interpretación de Imágenes: PB L1 frente a ESP L1.

Si bien en los dos casos la imagen que hemos llamado 'amigable' podría haber influido con algunos dibujos, sobre todo de los ítems con el rasgo [+animado], nos preguntamos si la diferencia entre los dos grupos puede deberse a que el valor afectivo del diminutivo tenga más fuerza en PB que en ESP y, por lo tanto, el tamaño que reflejaría el abarque sobre todo el compuesto no influye en la selección de la imagen en el caso de los hablantes de PB.

Vamos a ver ahora si estos patrones se reproducen en la prueba AJT, en la que contamos además con ejemplos no solo de derivación, sino también de flexión.

\subsection{Prueba de Juicios de Aceptabilidad: ESP y PB 4.4.1. Participantes}

Esta prueba la completaron un grupo de 66 hablantes nativos de español peninsular, todos adultos y con educación universitaria y con profesiones no ligadas al estudio o a la enseñanza de lenguas o de la traducción, y un grupo de 59 hablantes nativos de portugués brasileño, también adultos y con las mismas características profesionales que el grupo ESP. 


\subsubsection{Diseño de la prueba AJT}

La prueba se administró a través de la red. Se les presentaron ocho tipos de compuestos flexionados o con sufijos derivativos como los que figuran en las tablas 1 y 2 , y se les pidió que dieran un valor a cada ítem según una escala Likert de cinco puntos.

\begin{tabular}{lccc}
\hline \multicolumn{4}{c}{ TABLA 1. ESP. PRUEBA DE JUICIOS DE ACEPTABILIDAD } \\
\hline & CLASE DE COMPUESTO & FLEXIÓN & DERIVACIÓN \\
\hline $\mathbf{N N}$ & carta bomba & - & -- \\
\hline $\mathbf{x N}$ & afijo en el núcleo & cartas bomba & cartita bomba \\
\hline $\mathbf{N x}$ & afijo en el modificador & carta bombas & carta bombita \\
\hline $\mathbf{x x}$ & afijo en ambos Ns & cartas bombas & cartita bombita \\
\hline
\end{tabular}

\begin{tabular}{lccc}
\hline \multicolumn{4}{c}{ TABLA 2. PB. PRUEBA DE JUICIOS DE ACEPTABILIDAD } \\
\hline & CLASE DE COMPUESTO & FLEXIÓN & DERIVACIÓN \\
\hline $\mathbf{N N}$ & carta bomba & -- & -- \\
\hline $\mathbf{x N}$ & afijo en el núcleo & cartas-bomba & cartinha-bomba \\
\hline $\mathbf{N x}$ & afijo en el modificador & carta-bombas & carta-bombinba \\
\hline $\mathbf{x x}$ & afijo en ambos Ns & cartas-bombas & cartinha-bombinha \\
\hline
\end{tabular}

\subsubsection{Resultados. Grupo ESP L1 y Grupo PB L1}

En los gráficos 4 y 5 se muestran los resultados para la flexión y la derivación de ambos grupos. El símbolo x muestra dónde está el afijo, es decir, a la izquierda, en el núcleo $(\mathrm{xN})$, a la derecha, en el modificador $(\mathrm{Nx})$ o en los dos nombres del compuesto $(\mathrm{xx})$.

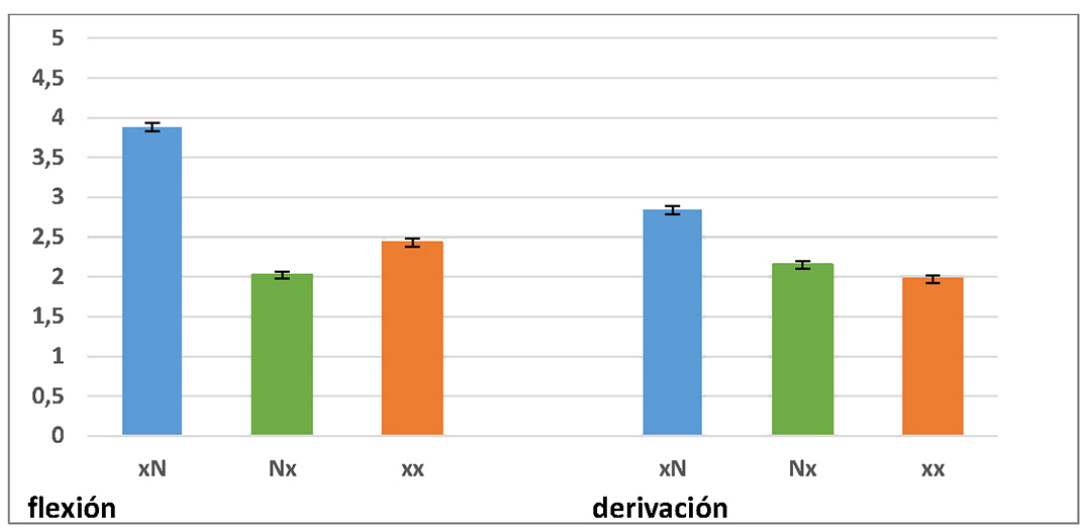

Gráfico 4. ESP L1. Prueba de Juicios de Aceptabilidad. 
Como puede observarse en el gráfico 4, los hablantes de español L1 prefieren los compuestos N-N que tienen el sufijo flexivo y el sufijo derivativo en el núcleo. En otras palabras, parece claro que son sensibles a la direccionalidad del núcleo. Como puede verse en el gráfico y también en la tabla 3, el plural en el modificador es la opción más rechazada en la morfología flexiva: la opción claramente agramatical.

\begin{tabular}{ccc}
\hline \multicolumn{2}{c}{ TABLA 3. ESP L1. ESCALA DE PREFERENCIA EN LA PRUEBA AJT } \\
\hline PREFERENCIA & NN + FLEXIÓN & NN + DERIVACIÓN \\
\hline$\# 1$ & $-s$ en el núcleo & -ito en el núcleo \\
\hline 2 & $-s$ en ambos Ns & -ito en el modificador \\
\hline$\# 3$ & $-s$ en el modificador & -ito en ambos Ns \\
\hline
\end{tabular}

En la morfología derivativa, la opción más rechazada es la que lleva -ito en los dos nombres del compuesto (la doble sufijación), lo cual parece indicar que la redundancia de -ito produce un rechazo más fuerte que la doble fijación de plural. Es posible que eso se deba a que el diminutivo tiene más significado.

Los hablantes del grupo de $\mathrm{PB}$, como podemos observar en el gráfico 5 y en la tabla 4, tienen exactamente las mismas preferencias que los hablantes del grupo de ESP, ya que la primera opción es también la de los compuestos con el afijo flexivo y derivativo en el núcleo, lo cual también nos permite afirmar que los hablantes nativos de PB L1 son sensibles a la direccionalidad del núcleo.

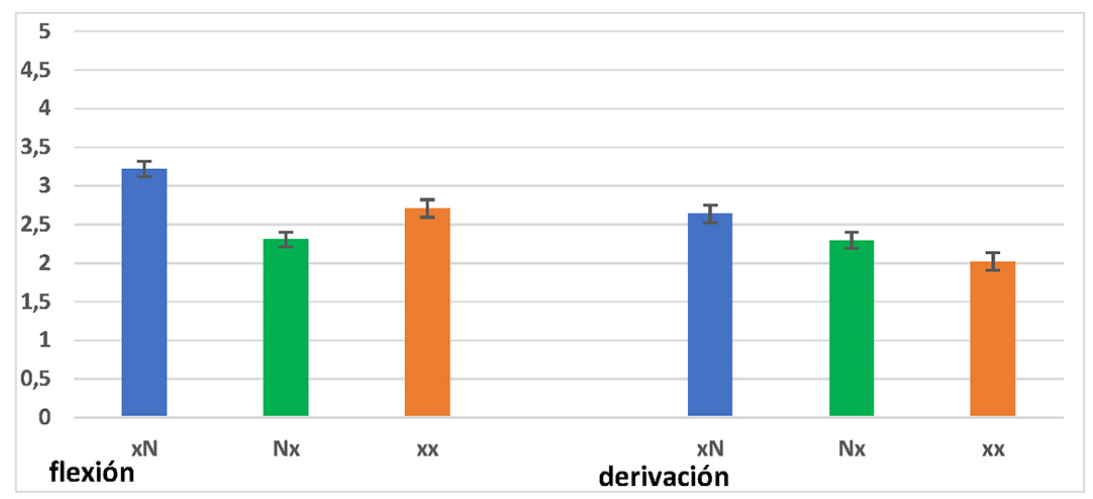

Gráfico 5. PB L1. Prueba de Juicios de Aceptabilidad

Coinciden también con los hablantes de ESP en que la opción más rechazable en la morfología flexiva es la que lleva el plural en el modificador, la que, como vimos en la sección 2 , se considera claramente agramatical. 


\begin{tabular}{ccc}
\hline \multicolumn{2}{c}{ TABLA 4. PB L1. ESCALA DE PREFERENCIA EN LA PRUEBA AJT } \\
\hline PREFERENCIA & NN + FLEXIóN & NN + DERIVACIÓN \\
\hline 1 & $-s$ en el núcleo & -ito en el núcleo \\
\hline 2 & $-s$ en ambos Ns & -ito en el modificador \\
\hline 33 & $-s$ en el modificador & -ito en ambos Ns \\
\hline
\end{tabular}

También la doble sufijación en la morfología derivativa (-inho en los dos sustantivos del compuesto) fue la opción más rechazada y, en este caso, no es una tendencia con respecto a la sufijación en el modificador, sino una diferencia estadísticamente significativa. De nuevo nos encontramos ante un efecto más fuerte de la redundancia con -inho que con la -s del plural.

\section{CONCLUSIONES}

Podemos concluir que, por lo que se refiere a la direccionalidad del núcleo, los nativos de ESP y de PB tratan la morfología flexiva y la derivativa de la misma forma en el caso de la prueba AJT. De hecho, en esta prueba los hablantes de ESP y de PB se comportan exactamente igual.

En la prueba PIT ninguno muestra una interpretación según la cual el afijo en el modificador pueda tener abarque sobre todo el compuesto, es decir, aquí tampoco hay evidencia de 'promiscuidad' del sufijo derivativo (Liceras \& Klassen, 2019). Sin embargo, de la prueba PIT, pero no de la prueba AJT, parece desprenderse que para los hablantes de $\mathrm{PB}$ el abarque solo afecta al sustantivo al que va ligado el sufijo, tanto si va ligado al núcleo como si va ligado al modificador. Esta discrepancia entre las dos pruebas puede indicar que la prueba PIT condiciona los resultados. Por lo tanto, parece claro que necesitamos pruebas que nos permitan eliminar el efecto de las imágenes y que nos permitan constatar la aportación semántica que hace el sufijo al compuesto o a sus partes. Es posible que con una prueba online que mida el tiempo de reacción podamos medir la fuerza que tiene la interpretación por defecto (tamaño) del sufijo diminutivo en ambas lenguas.

También es importante señalar que la interpretación de los N-N con el rasgo [-animado] como carta bomba o coche cama es más clara que la de los compuestos N-N con el rasgo [+animado] como hombre lobo o mujer pulpo, ya que las opciones de los participantes son más categóricas (hay menos variabilidad). Creemos que esto puede deberse a que el rasgo [+/-animado] está claramente ligado al rasgo [+/separable], y esto implica que es más fácil conceptualizar los compuestos que constan de dos entidades separables (una carta y una bomba, un coche y una cama) que los compuestos que constan de dos entidades o elementos que aparecen entrelazados como es el caso de un hombre y un lobo o una mujer y un pulpo, que no son dos entes sino un ente con rasgos o partes de ambos. De hecho, en la prueba PIT los hemos representado o con cabeza de lobo y cuerpo de hombre o con cuerpo de mujer y brazos de pulpo respectivamente. Si bien en los datos hemos notado que en los compuestos con rasgo [-animado] es más clara la tendencia a que se prefiera 
que el sufijo a la izquierda marque la direccionalidad del núcleo, las diferencias no son significativas y, de hecho, como la prueba no se ha diseñado aislando animacidad de separabilidad, no podemos llegar a conclusiones claras sobre el papel de la animacidad. Hemos de precisar también que, aunque en su momento lo intentamos, no está claro que se puedan encontrar compuestos N-N no animados «inseparables» (tal vez sofá cama pero no muchos más) y, desde luego, no tenemos claro que se puedan conceptualizar compuestos N-N con el rasgo [+animado] y que sean «separables». Este es un tema que esperamos que sea objeto de investigación tanto por parte de los lingüistas como de los psicolingüistas.

Finalmente, nos gustaría proponer que, además de los compuestos N-N, los llamados compuestos 'deverbales' o 'exocéntricos' pueden ser también de interés para investigar cuestiones de ambigüedad sobre el abarque de los sufijos evaluativos. Es un área sin explorar y no siempre parece tan clara la relación de tamaño que se muestra entre el abrelatas de (20)a y el de (20)b, ya que podríamos plantear que el diminutivo solo tiene abarque sobre el sustantivo del compuesto y no sobre el tema verbal, de forma que no sea pequeño, sino que podría tener el mismo tamaño pero estar destinado a abrir latas pequeñas.

(20) a abrelatas

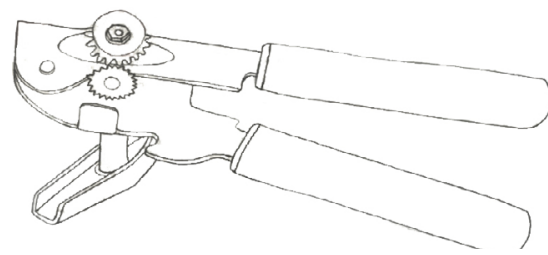

(20) b abrelatitas

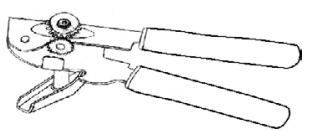

Esta es una opción que, cuando se les presenta a los nativos, no se elige como la preferida. Sin embargo, y dada la relación semántica que se establece entre el verbo y el sustantivo en los muchos compuestos deverbales que están atestiguados y en los que se pueden 'crear' e 'interpretar', es posible que dichas interpretaciones no impliquen siempre la opción que podemos llamar no marcada o por defecto, es decir, la de que el diminutivo tiene abarque sobre todo el compuesto. 


\section{BIBLIOGRAFÍA}

Allen, Margaret (1978): Morphological Investigations, PhD. dissertation, University of Connecticut.

Bermúdez-Otero, R. (2007): «Spanish pseudo-plurals: Phonological cues in the acquisition of a syntax-morphology mismatch». Proceedings of the British Academy 145: 231-269.

BooIJ, Geert (1996): «Inherent versus contextual inflection and the split morphology hypothesis», en Geert Booij y Jaap Van Marle (eds.), Yearbook of Morphology 1995. Dordrecht: Kluwer, 1-16.

Bybee, Joan (1985): Morphology: A Study of the relation between meaning and form, Amsterdam: John Benjamins.

Сномкку, Noam (1981): Lectures on Government and Binding, Berlin: Mouton de Gruyter.

Сномsкy, Noam y Howard Lasnik (1993): «Principles and Parameters Theory», en Armin von Stechow, Joachim Jacobs, Wolfgang Sternefeld y Theo Vennemann (eds.), Syntax: An International Handbook of Contemporary Research, Berlin: de Gruyter. Reimpreso en Chomsky (1995): The minimalist program, chapter.1. Chomsky, 13-127. Cambridge MA: MIT Press.

Cinque, Guglielmo (2005): «Deriving Greenberg's Universal 20 and its exceptions», Linguistic Inquiry 36 (3): 315-332.

Klassen, Rachel, Isabel Contro Castaldo y Juana M. Liceras (2018): «Native and non-native Brazilian Portuguese grammars through the lens of derivational morphology in compounding», Romance Turn 9, Universidad de Bucarest, 30 de agosto-1 de septiembre.

LiCERAS, Juana M. (2004): «La adquisición del lenguaje no nativo y la 'manipulación del input': ¿Podemos crear desencadenantes pedagógicos?», en Christoph Ehlers y Anton Haidl Dietlmeier (eds.), El alemán en España: motivaciones y perspectives, actas del Congreso III de FAGE (Federación de Asociaciones de Germanistas de España), Málaga, 1-20.

Liceras, Juana M. y Rachel KLASSEN (2016): «The representation of headedness in the mind of non-native Spanish speakers: evidence from compounding and derivation", EUROSLA 26, Universidad de Jyväskylä, Finlandia, 24-27 de agosto.

Liceras, Juana M. y Rachel Klassen (2019): “Compounding and derivation: On the 'promiscuity' of derivational affixes", Linguistic Approaches to Bilingualism 9: 42-72.

Liceras, Juana M., Lourdes Díaz y Terhi SalomaA-Robertson (2002): «The Compounding Parameter and the word marker hypothesis: accounting for the acquisition of Spanish N-N compounding», en Ana Teresa Pérez-Leroux y Juana M. Liceras (eds.), The Acquisition of Spanish Morphosyntax: The L1/L2 Connection, Dordrecht: Kluwer, 209-237.

Pomerleau, Julie (2001): La adquisición del español en el aula: los compuestos nominales de los franceses de Canadá. tesis de M.A., Universitat de Barcelona.

Salomaa-Robertson, Terhi (2000): L2 Acquistion of Spanish compounds by native speakers of Finnish, tesis de M.A., University of Ottawa.

SNyder, William (1995): Language Acquisition and Language Variation: The Role of Morphology, tesis doctoral MIT. Distribuida por MIT Working Papers in Linguistics.

SNYDER, William (2001): «On the nature of syntactic variation: Evidence from complex predicates and complex word-formation", Language 77:324-342.

Snyder, William (2007): Child Language: The Parametric Approach, Oxford, UK: Oxford University Press.

ZwICKy, Arnold (1985): «Heads», Journal of Linguistics 21(1): 1-29. 
